Fernández-Jiménez, M.A., Mena-Rodríguez, E. y Tójar-Hurtado, J.C. (2017). Funciones de la tutoría en e-learning: Estudio mixto de los roles del tutor online. Revista de Investigación Educativa, 35(2), 409-426

DOI: http://dx.doi.org/10.6018/rie.35.2.273271

\title{
Funciones de la tutoría en e-learning: Estudio mixto de los roles del tutor online
}

\author{
Tutoring functions in e-learning: \\ Mixed study of the roles of the online tutor
}

\author{
Miguel-Ángel Fernández-Jiménez, Esther Mena-Rodríguez y Juan-Carlos Tójar-Hurtado \\ Universidad de Málaga (España)
}

\begin{abstract}
Resumen
Desde que en 1924 el Pressey Testing Machine apareció en la historia de la educación hasta el 2013 donde los MOOC están presentes a nivel mundial, el e-learning ha sufrido una evolución constante. Ello se ha debido en parte a que es una nueva forma de aprendizaje adaptado a las necesidades de una sociedad cambiante, en la que los trabajadores necesitan capacitarse fuera de su horario laboral. De esta manera, la investigación en los procesos implícitos en el e-learning se hace imprescindible de cara a la mejora de la calidad de esta modalidad de formación. Por ello el objetivo de esta investigación se dirigió a 1) analizar las funciones que los formadores llevan a cabo en sus acciones formativas $y, 2$ ) investigar cómo aprecian los estudiantes esas funciones. La metodología aplicada es un diseño mixto para adecuarse lo mejor posible a los objetivos de la investigación. Se ha utilizado una muestra de 707 estudiantes y otra muestra de los tutores de 29 cursos, ambas pertenecientes a un proyecto de formación continua de trabajadores de pequeñas y medianas empresas (PYMES). De los resultados obtenidos es posible conocer las funciones que los tutores han llevado a cabo y, a partir de ahí, construir algunos "marcos teóricos" que permitan comprender las relaciones entre categorías y dimensiones que subyacen a este tipo de formación. Del mismo modo, se han podido determinar diferentes tipologías de tutores dependiendo de los roles que han desempeñado.

Palabras clave: e-learning; tutor e-learning; teleformación; funciones del tutor e-learning.

Correspondencia Miguel Ángel Fernández Jiménez, mafjimenez@uma.es, Facultad de Ciencias de la Educación, Bulevar Louis Pasteur s/n, 29071, Málaga, España.
\end{abstract}




\begin{abstract}
Since in 1924 the Pressey Testing Machine appeared in the history of education until 2013 where MOOCs are present worldwide, e-learning has evolved steadily. This has been partly because it is a new way of learning adapted to the needs of a changing society, where workers need to be trained outside their working hours. Along these lines, the research in the implicit processes in the e-learning becomes essential in order to improve the quality of this modality of training. Therefore, the objective of this research was to 1) analyze the functions that trainers carry out in their training actions and 2) investigate student's appreciation of these functions. The methodology applied was a mixed design to best fit the objectives of the research. A sample of 707 students and another sample of tutors of 29 courses were used, both belonging to a continuous training project of workers of small and medium-sized enterprises (SMES), From the results it is possible to know the functions that tutors have been carring out and, from there, build some "theoretical framework" for understanding the relationships between categories and dimensions underlying this kind of training. Likewise, it has been possible to determine different typologies of tutors depending on the roles they have played.

Keywords: e-learning; online tutor; online distance education; functions of e-learning tutor.
\end{abstract}

\title{
Introducción
}

La introducción de las TIC en la Educación supone un replanteamiento en lo referente a los elementos que intervienen en el proceso de enseñanza-aprendizaje. El e-learning cada vez más, va ganando terreno en el ámbito de la formación, tanto por su eficiencia como por el incremento de estudios que día a día ven la luz con el objetivo de mejorar el proceso de E-A (p. ej. Cabero, 2013; Llorente, 2006).

En esta nueva situación cambiante y dinámica, se deben replantear los métodos, estrategias e instrumentos a utilizar en la enseñanza, ya que la formación continua que precisan las personas no será única ni puntual a lo largo de sus vidas (p. ej. Volles, 2016).

De la misma forma, las TIC han ido adquiriendo un papel más relevante, permitiendo realizar los procesos de aprendizaje de manera más eficiente, pudiendo así compatibilizar las variables espacio y tiempo (Fandos \& Cano, 2013; Fernández Robles \& Cabero, 2016). De ahí que el e-learning sea la modalidad más utilizada en la formación continua de trabajadores debido a las ventajas que presenta (Cabero, 2013; Fernández Robles \& Cabero, 2016).

Ahora bien, a pesar de estos avances y cambios en la formación, y en el e-learning en particular, no se puede pensar que esta modalidad formativa garantice una mayor calidad, un aprendizaje más rápido y más eficaz por sí sola. En la modalidad e-learning el papel del tutor es clave (Cabero, 2013; Cabero \& Llorente, 2010; Curci, 2014), y supone un cambio en el rol que desempeñaba éste, pasando de ser el "sabio en la tarima" a ser el "guía que acompaña". En este sentido, se pueden distinguir al menos tres funciones principales inherentes al rol de guía: como diseñador del trabajo de los estudiantes, como facilitador del proceso de aprendizaje y como mentor (Reigeluth, 2012).

La investigación que se presenta aborda una doble perspectiva: por un lado, la introducción de las TIC en la educación y la formación, como un aspecto relevante y 
actual; y por otro lado, la necesidad de mejorar la calidad del e-learning en el ámbito de la formación de trabajadores.

A pesar de que el e-learning cuenta con un gran número de ventajas, uno de los principales problemas que presenta es el alto índice de abandono de estudiantes si lo comparamos con otras modalidades de formación (Aballay, Aciar, González, \& Collazos, 2016; Fernández-Jiménez, Tójar-Hurtado, \& Mena-Rodríguez, 2013; Fidalgo, Sein-Echaluce, Lerís, \& Castaneda, 2013; García Teske, 2007; Rodríguez, 2014; Sánchez López \& Gallego, 2014). Este abandono se acentúa en los cursos que son realizados por trabajadores.

Debido lo expuesto anteriormente, este trabajo quiere contribuir a mejorar la calidad de las acciones formativas que se desarrollan en este ámbito de la formación. Para ello, se parte de analizar cuáles son las funciones que realizan los tutores e-learning durante las acciones formativas en las que participan, y cómo perciben esas funciones los estudiantes.

La propuesta que realizó Llorente (2005) y que ha sido considerada por otros muchos autores (p. ej. Fernández-Jiménez et al., 2013; Sánchez López \& Gallego, 2014; Picón, 2013) sobre las distintas funciones que debe desempeñar un teletutor, se ha tomado como modelo teórico y punto de partida de esta investigación. Esta autora propuso cinco funciones (Fernández-Jiménez et al., 2013, p. 87):

- Función Académica: Referente al dominio de los contenidos, actividades, diagnóstico y evaluación formativa de los estudiantes, así como las habilidades para organizar las actividades.

- Función Técnica: A través de la cual se pretende asegurar el dominio de los estudiantes sobre las herramientas disponibles en el entorno virtual.

- Función Orientadora: Que tiene como finalidad la de tener la habilidad suficiente para planificar, estructurar y saber establecer las normas de funcionamiento, así como programar en el tiempo y poner en marcha las distintas acciones formativas.

- Función Social: Mediante la cual se pretende evitar los sentimientos de aislamiento, pérdida o falta de motivación del alumnado en esta modalidad formativa.

- Función Organizativa: Hace referencia a la labor de guiar y asesorar en cuestiones organizativas al alumnado durante el desarrollo de las acciones formativas.

\section{Método}

\section{Objetivos}

Los objetivos planteados en esta investigación fueron:

1. Diseñar, construir y validar un cuestionario para los estudiantes trabajadores de PYMES, y autónomos que realizan cursos de formación, con la intención de estudiar las funciones de los tutores durante las acciones formativas. 
2. Conocer las percepciones que tenían los estudiantes sobre las distintas dimensiones o funciones que empleó cada tutor en las distintas acciones formativas.

3. Identificar y comprender las dimensiones y funciones puestas en práctica por los tutores e-learning del estudio durante las acciones formativas a través del análisis del entorno virtual de aprendizaje y conocer si existían diferencias entre ellos.

4. Comprender las percepciones de los tutores y analizar las diversas formas de concebir la tutoría online de cursos de formación.

5. Recabar las opiniones de los tutores sobre cuáles fueron las funciones y tareas que consideraban como más importantes en la tutorización online.

\section{Población y Muestra}

Para la investigación se tomaron dos muestras: por un lado, 707 estudiantes, pertenecientes a un proyecto de formación continua en el que se formaba a trabajadores de toda España, en 29 cursos en la modalidad e-learning; y por otro, los tutores que guiaron dichos cursos.

Ante la imposibilidad de realizar un muestreo aleatorio efectivo que garantizara la representatividad de la muestra, se optó por realizar un muestreo no probabilístico de tipo incidental. Se solicitó para ello la participación voluntaria del alumnado del proyecto través de un cuestionario online, y de los tutores a través de una entrevista semi estructurada con una posterior solicitud de ampliación de información por escrito.

\section{Procedimiento de recogida de datos}

La metodología empleada en esta investigación aplicó técnicas cuantitativas y cualitativas, buscando en cada momento, el método que mejor se adaptara a los objetivos planteados inicialmente.

En un primer momento se utilizó un diseño de investigación de tipo encuesta o "survey". Para ello se elaboró un cuestionario que permitió describir actitudes, creencias y opiniones de los estudiantes sobre la tutoría recibida en los cursos.

En la segunda fase de la investigación se analizó la plataforma virtual utilizada por los tutores, estudiando así las variables de estudio propuestas inicialmente. Para ello se confeccionó una hoja de registro, en la que se fueron recogiendo si los tutores realizaban o no las tareas que se proponían como variables de estudio.

La última fase se orientó desde una perspectiva cualitativa con la finalidad de profundizar en algunas de las variables de estudio. Para ello se analizó la opinión de los tutores mediante entrevistas. Con dichas entrevistas se pretendía obtener una amplia variedad de perspectivas y visiones conceptuales, que ayudara a entender, con mayor profundidad, algunas de las dimensiones claves en el complejo proceso de tutorización.

Esta fase se completó con una ampliación de información a través de una ficha de registro a los tutores entrevistados. Por medio de ella se pidió su opinión sobre cómo se podría mejorar, desde su punto de vista y su experiencia profesional, el proceso de tutorización en la formación e-learning. 


\section{Instrumento y técnicas de recogida de datos}

El cuestionario elaborado para los estudiantes se dividió en varios bloques (Fernández-Jiménez et al., 2013):

En el primer bloque se recogía información referente a: motivo de solicitud del curso, causas por las que dejaría el curso y cumplimiento con las expectativas planteadas por el estudiante antes del comienzo del curso.

En la segunda parte se concentraban los ítems referentes a las variables que fueron determinadas a partir de las categorías constituidas por las cinco funciones fundamentales de estudio (Llorente, 2005). Dichas variables se presentan en la tabla 1.

Tabla 1

Categorías constituidas por las cinco funciones que se incluyeron en el cuestionario extraídas de Llorente (2005)

Categorías (Funciones)

Académica

\section{Variables de estudio}

Intentar mantener flexibilidad.

Establecimiento de feedback a los estudiantes.

Animación a la participación.

Respuesta rápida hacia el alumnado y las actividades presentadas.

Diseño de las actividades y situaciones de aprendizaje de acuerdo a los objetivos planteados.

Creación, animación y dinamización de la participación en los foros disponibles.

Adaptación de los contenidos a los objetivos propuestos.

Resolución de dudas en máximo de 48 horas.

Técnica

Aseguramiento del conocimiento y habilidad en el manejo del entorno virtual.

Apoyo técnico.

Consecución de la adaptación del alumnado al entorno virtual.

Aseguramiento del dominio de las herramientas asincrónicas y sincrónicas del entorno virtual.

Orientadora

Asesoramiento sobre el trabajo y la calidad del mismo.

Orientación e información a los estudiantes sobre sus progresos.

Aseguramiento de la utilización de las herramientas con las que cuenta la plataforma como forma de comunicación con el tutor.

Social

Control y evitación de la sensación de soledad.

Organizativa
Establecimiento y explicación de las normas de funcionamiento. 
Previo a la aplicación del instrumento, se realizó un estudio de las características psicométricas del mismo y se recogieron evidencias sobre la fiabilidad y la validez de la medida que proporcionaba. Fue validado por 6 expertos en metodología de investigación, pertenecientes a varios departamentos de Educación de la Universidad de Málaga sin relación con la investigación. Asimismo, se comprobó que presentaba una consistencia interna aceptable (a de Cronbach de .885). Para analizar la validez de constructo se realizó un análisis factorial categorial de componentes principales (CATPCA), teniendo en cuenta la naturaleza ordinal de la escala Likert utilizada. El resultado fue satisfactorio. Se obtuvieron 6 componentes principales o factores (que explicaban el $62.02 \%$ de la varianza total), coherentes con las funciones tutoriales ("función tutorial y características del tutor", "herramientas de comunicación y contacto con el tutor a través de e-mail, foro y chat", "explicaciones del tutor y resolución de problemas de la plataforma”, "autonomía del alumnado", "apoyo con otros materiales (manual o guía)" y "facilitación en la temporalización de las tareas y actividades").

La segunda fase de la investigación se apoyó en un análisis del entorno virtual que se emplea para la impartición de los cursos a los que se hace mención en este estudio.

A través de este análisis se pretendía recabar información acerca de algunas variables de estudio que se podían estudiar directamente en el entorno virtual a través de la huella dejada por los tutores. Las variables estudiadas se detallan a continuación en la tabla 2.

Tabla 2

Variables estudiadas a través del entorno virtual

Categorías (Funciones) Variables de estudio

Académica Dar información, extender, clarificar y explicar los contenidos presentados.

Resumir los debates en grupo de participación de los estudiantes extrayendo conclusiones.

Técnica Incorporar materiales al entorno de formación.

Ofrecer una guía de estudio o manual de usuario del entorno.

Orientadora

Facilitar técnicas de trabajo intelectual. Motivar a los estudiantes.

Ser guía y orientador de los estudiantes.

Social

Dar la bienvenida.

Incitar a los estudiantes a que amplíen y desarrollen argumentos presentados por los compañeros en foros de discusión y en actividades grupales.

Integrar y conducir las intervenciones.

Animar y estimular la participación.

Proponer actividades para facilitar el conocimiento entre los participantes. 
Organizativa

Establecer y explicar las normas de funcionamiento.

Organizar a los estudiantes para realizar actividades grupales.

Establecer calendario.

En la tercera fase de la investigación se realizaron entrevistas a los tutores que desempeñaron su labor en las 29 acciones formativas del proyecto. A través de esta técnica se pretendió conocer la opinión y perspectiva de estos sobre algunas de las variables de estudios que aparecen detalladas en la tabla 3.

Tabla 3

Variables estudiadas a través de las entrevistas a tutores

\section{Categorías (Funciones)}

Académica

Técnica

Orientadora

Social

Organizativa

\section{Variables de estudio}

Asegurarse de que los estudiantes están alcanzado un nivel adecuado.

Hacer valoraciones globales e individuales de las actividades realizadas.

Realizar actividades formativas.

Contacto estricto con el administrador del sistema.

Facilitar técnicas de trabajo intelectual.

Asegurarse de que el ritmo de trabajo de los estudiantes es el adecuado.

Motivar a los estudiantes.

Ser guía y orientador del alumnado.

Animar y estimular la participación.

Mantener contacto con el resto de equipo docente del curso.

Establecer estructuras en la comunicación online.

Organizar y saber realizar las tareas administrativas que exija el proyecto formativo.

\section{Análisis de datos}

En la primera de las fases se llevó a cabo una serie de análisis que se dividió en dos partes principales. La primera de ellas consistió en un análisis de tipo descriptivo en el que se analizaron las variables tenidas en cuenta en el cuestionario. Se calcularon 
las medias, desviaciones típicas y medianas de todos los ítems del cuestionario. Así mismo, se realizaron representaciones de distribuciones de frecuencias, gráficas de perfiles y de sectores, teniendo en cuenta las variables edad, género, nivel de estudios, contrato laboral, sector empresarial al que pertenecían los estudiantes, cursos y las provincias donde residían.

Posteriormente y para comprobar si existen o no diferencias en la percepción que tienen los estudiantes sobre los mejores tutores, se planteó un diseño multivariante. Como variable independiente se tomaron los distintos tutores participantes, y como dependientes cada una de las escalas del cuestionario, esto es, funciones técnica, académica, organizativa, orientadora y social. El diseño se abordó a través de un análisis multivariante de la varianza (MANOVA). Para probar la igualdad de las matrices de covarianzas se utilizó la prueba de Box, que nos ha permitido contrastar la hipótesis nula de que las matrices de covarianza observadas eran iguales en todos los grupos.

Se realizó también el contraste de Levene sobre la igualdad de las varianzas de error, para contrastar la hipótesis de que la varianza de error de la variable dependiente era igual a lo largo de todos los grupos. Tras la realización de los contrastes multivariados y univariados se procedió a la realización de comparaciones múltiples (pruebas post hoc) mediante la DHS de Tukey y Dunnett para determinar en qué escalas concretas se producían diferencias entre los distintos tutores.

Los análisis cuantitativos se han realizado con el paquete estadístico SPSS versión 20 para Windows.

La segunda fase consistió en un análisis descriptivo de la huella digital dejada por los tutores en el entorno virtual de formación. En ella se fueron comprobando y verificando si los tutores realizaban las tareas o no durante el curso, si existían diferencias entre ellos, y si esas diferencias eran debidas o no al azar.

En la tercera fase los análisis fueron de tipo cualitativo. Como unidades de análisis se tomaron las expresiones literales expresadas por los tutores en las entrevistas realizadas.

En un primer momento se elaboraron "nubes de palabras". Estrategia que tiene mucha popularidad en la actualidad en investigaciones en el campo de las Ciencias de la Educación (McNaught \& Lam, 2010; Perry, 2012). Para ello, se utilizó la aplicación Tagxedo.

Tras realizar la primera aproximación a los datos mediante la nube de palabras, se procedió a profundizar en los análisis mediante un proceso de categorización. Cada categoría establecida, se elaboró a través de una selección de las distintas unidades de análisis y de la realización de precategorías. Este paso se llevó a cabo de manera simultánea al de análisis y generación de conclusiones.

A toda la información recogida en las entrevistas y la posterior ampliación de información, se le aplicó un proceso de reducción y disposición de datos, así como de elaboración y verificación de conclusiones. Para ello se emplearon distintas técnicas analíticas mediante una escala creciente de abstracción, incluyendo análisis especulativo, clasificación y categorización, formación de categorías y subcategorías, modelos y tipologías, para que los datos elaborados fueran tomando cuerpo de teoría. 
Los procesos analíticos y de teorización mencionados anteriormente se usaron como instrumentos conceptuales de elaboración de los análisis, a través del empleo de matrices o tablas y de gráficas descriptivas y explicativas.

Para realizar los análisis cualitativos se utilizó la aplicación Atlas.ti 7.0.

\section{Resultados}

Los resultados descriptivos iniciales mostraron que uno de los principales motivos que motivó a los estudiantes a realizar la formación fue el de mejorar en su trabajo y mejorar su currículo personal. De manera que no realizaban la formación porque no tuvieran nada mejor que hacer, o porque alguien les convenciera para ello.

Entre las posibles causas de abandono de la formación por parte de éstos, estaría la falta de tiempo, pero no la abandonarían por tener un escaso dominio del entorno virtual de aprendizaje o informático, por estar poco motivado una vez comenzada la formación, por tener problemas de acceso a Internet o a un ordenador para poder llevar a cabo las acciones formativas o porque el tutor no les motivara e incentivara durante la realización de las mismas. No queda claro que una de las causas pudiera ser la de que no existiera una relación entre los objetivos planteados en la formación y las expectativas iniciales de los estudiantes.

Casi la totalidad de los estudiantes indican que los cursos realizados habían cubierto sus expectativas.

En la tabla 4 aparecen los resultados de los ítems del cuestionario que se mencionaron anteriormente. En ella aparecen las frecuencias de los ítems, la media, la desviación típica y la mediana.

Tabla 4

Resultados del cuestionario de estudiantes de la fase uno de la investigación

\begin{tabular}{|c|c|c|c|c|c|c|c|}
\hline Ítem del cuestionario & f1 & $\mathrm{f} 2$ & f3 & f4 & & Sx & Me \\
\hline $\begin{array}{l}\text { El motivo por el que he } \\
\text { solicitado el curso ha sido: } \\
\text { Para mejorar en mi trabajo }\end{array}$ & 0 & 32 & 219 & 456 & 3.60 & 0.58 & 4.00 \\
\hline $\begin{array}{l}\text { El motivo por el que he } \\
\text { solicitado el curso ha sido: } \\
\text { Para mejorar mi currículum } \\
\text { personal }\end{array}$ & 5 & 26 & 155 & 521 & 3.69 & 0.58 & 4.00 \\
\hline $\begin{array}{l}\text { El motivo por el que he } \\
\text { solicitado el curso ha sido: } \\
\text { Porque no tenía nada mejor } \\
\text { que hacer }\end{array}$ & 518 & 95 & 29 & 65 & 1.49 & 0.94 & 1.00 \\
\hline
\end{tabular}


El motivo por el que he solicitado el curso ha sido: Yo no quería hacerlo, pero me han convencido

Diría que la causa por la que dejaría el curso es: Falta de tiempo para realizar las tareas y/o estudiar los contenidos del curso

Diría que la causa por la que dejaría el curso es: Los contenidos no se correspon-

den con mis expectativas

Diría que la causa por la que dejaría el curso es: El tutor no me ha motivado ni incentivado lo suficiente para poder realizar el curso

Diría que la causa por la que dejaría el curso es: $\mathrm{He}$ empezado el curso y no me encuentro motivado haciéndolo

Diría que la causa por la que dejaría el curso es: Poco dominio de la plataforma virtual de formación

Diría que la causa por la que dejaría el curso es: Me cuesta mucho seguir el curso a través de Internet porque no domino la informática

Diría que la causa por la que dejaría el curso es: Problemas para tener acceso 390 145

$$
177
$$

$1.14 \quad 2.00$

313

170

126

98

2.01

$1.09 \quad 2.00$

316

143

$$
112
$$

136

2.10

$1.17 \quad 2.00$

438

129

43

97

1.72

$1.07 \quad 1.00$

a Internet o a un ordenador para poder hacerlo

Puedo decir que el curso está cubriendo mis expectativas y era lo que esperaba 
Los resultados descriptivos sobre los ítems relacionados con las funciones de los tutores muestran que las funciones organizativa, académica y orientadora, por este orden, son las variables más valoradas por los estudiantes. En cambio, las funciones con menor puntuación son la técnica y la social.

En la tabla 5 aparecen los resultados de los ítems relacionados con las funciones de los tutores del cuestionario que se mencionaron anteriormente. En ella aparecen las medias, desviaciones típicas y medianas de cada una de las funciones.

Tabla 5

Resultados descriptivos sobre los items relacionados con las funciones de los tutores

\begin{tabular}{lccc}
\hline Categorías (Funciones) & & Sx & Me \\
\hline Académica & 2.76 & 0.57 & 2.71 \\
Técnica & 3.46 & 0.55 & 3.63 \\
Orientadora & 3.49 & 0.68 & 4.00 \\
Social & 3.37 & 0.71 & 3.50 \\
Organizativa & 2.76 & 0.64 & 2.75 \\
\hline
\end{tabular}

Tras realizar el estudio en el entorno virtual, se comprobó que en algunas de las variables, el valor fue constante, es decir, o lo hicieron todos los tutores o, por el contrario, ninguno puso en práctica la actividad.

Las variables que los tutores realizaron en su totalidad son las siguientes:

- "Incorporar materiales al entorno de formación", es decir, se subieron a la plataforma artículos y materiales adicionales al temario que ya se encontraba disponible para el alumnado.

- "Ofrecer una guía de estudio o manual de usuario del entorno" para que pudieran manejarse por ella con el mínimo problema.

- "Establecer calendario del curso". El propio entorno virtual ya tenía configurado un bloque en el que aparecía el calendario. Fue el tutor el que definió y marcó, en el total de los casos, los eventos más importantes y significativos del curso.

- "Dar la bienvenida a los estudiantes" a través de alguna de las herramientas con las que contaba el entorno virtual. La totalidad de los tutores la realizaron a través de un foro.

- "Proponer actividades para facilitar el conocimiento entre los participantes". Esta tarea la hicieron todos los tutores igualmente a través de los foros.

Por el contrario, ninguno de los tutores puso en práctica:

- "Organizar a los estudiantes para realizar actividades grupales", ya que en ningún momento se llevaron a cabo ningún tipo de actividades grupales. 
- "Facilitar técnicas de trabajo intelectual para el estudio en la red". Tampoco se les facilitó al alumnado ningún tipo de estrategias ni un manual que les proporcionara información o asesoramiento sobre técnicas de trabajo intelectual.

$\bullet$

- En el resto de variables si hubo diferencias significativas en cuanto a los tutores:

- "Dar información, extender, clarificar y explicar los contenidos presentados".

- "Resumir debates sacando conclusiones".

- "Establecer y explicar normas de funcionamiento dentro del entorno virtual de aprendizaje".

- "Motivar al alumnado para el trabajo".

- "Ser guía y orientador del estudiante".

- "Incitar a desarrollar y ampliar argumentos en los foros".

- "Integrar y conducir las reuniones".

- "Animar a la participación".

En la tabla 6 se muestran las variables en las que hubo diferencias significativas entre los distintos tutores con los porcentajes de éstos que pusieron en práctica cada una de esas variables.

Tabla 6

Variables en las que hubo diferencias significativas entre tutores con los porcentajes de éstos que pusieron en práctica cada una de esas variables

\begin{tabular}{lc}
\hline \multicolumn{1}{c}{ Variables } & $\begin{array}{c}\text { \% de tutores que pusieron en } \\
\text { práctica la variable }\end{array}$ \\
\hline $\begin{array}{l}\text { Dar información, extender, clarificar y explicar los } \\
\text { contenidos presentados }\end{array}$ & $39.04 \%$ \\
$\begin{array}{l}\text { Resumir debates sacando conclusiones } \\
\text { Establecer y explicar normas de funcionamiento den- } \\
\text { tro del entorno virtual de aprendizaje }\end{array}$ & $25.32 \%$ \\
Motivar al alumnado para el trabajo & $39.04 \%$ \\
Ser guía y orientador del estudiante & $31.68 \%$ \\
Incitar a desarrollar y ampliar argumentos en los foros & $31.68 \%$ \\
Integrar y conducir las reuniones & $25.32 \%$ \\
Animar a la participación & $25.32 \%$ \\
\hline
\end{tabular}

Por otra parte, para comprobar si estas diferencias obtenidas en los valores entre los distintos tutores podían ser atribuibles o no al azar, se realizó la prueba no paramétrica $c^{2}$ de Pearson para aquellas variables o tareas que no tenían un valor constante (todos o ninguno de los tutores las pusieron o prácticas). El análisis de los datos mos- 
tró que, $p \leq .005$, en todos los casos se puede concluir que las tareas no se realizaron de la misma forma entre los distintos tutores y esa diferencia además no podía ser explicada por el azar.

Tras el análisis de todo el material textual con Atlas.ti de las trascripciones de las entrevistas a tutores, se seleccionaron 245 segmentos de texto o citas literales. Sobre dichas citas se elaboraron 212 códigos organizados en 11 familias. Como algunas de las citas literales fueron codificadas y recodificadas varias veces, el número total de citas x códigos ascendió a 408 unidades.

Tras un reagrupamiento de las familias de códigos (recategorización) se elaboraron 3 macro categorías:

Una primera relacionada con los "Recursos del tutor", que contiene las familias de "Materiales", "Herramientas de comunicación”, "Actividades y tareas", "Aspectos técnicos", "Foros", "Contenidos" y "Chat". Esta macro categoría se refería a los recursos que utilizó el tutor en su labor de tutorización.

Una segunda relativa a las "Funciones y Tareas del tutor", que incluía las familias de "Tareas del tutor", "Atención, tutoría y apoyo", "Organización y Planificación” y "Evaluación, seguimiento y control", que haría referencia a todas aquellas actividades, labores u ocupaciones del tutor desarrollando sus funciones.

Y una tercera y última, que contiene una sola familia: "Características del alumnado", que se han mantenido con el mismo nombre, y que reuniría todos aquellos rasgos que caracterizan a los estudiantes que realizan este tipo de cursos.

Tras realizar el análisis de los documentos con información adicional aportados por los tutores, se construyeron 4 macro categorías:

En primer lugar la macro categoría "Funciones del tutor", a partir de las opiniones de los tutores y de las categorías elaboradas que construyen el rol del tutor a partir de sus funciones. Dentro de esta macro categoría se encontrarían las "Funciones pedagógicas" que se refieren al control y seguimiento que tiene que hacer el tutor de los estudiantes, relacionar los contenidos teóricos con la práctica profesional y planificar y organizar el trabajo a realizar, las "Funciones sociales y dinamizadoras" que incluyen la necesaria presentación e introducción del curso y el acompañamiento durante el curso y en la que el tutor debe fomentar la interacción entre el estudiante y él mismo y entre aquel y el resto de los estudiantes, las "Funciones de gestión y organización" que hacen mención al cumplimiento de obligaciones por ambas partes, la evaluación sumativa del estudiante, la confección y el seguimiento del calendario y a la planificación y, por último, las "Funciones Técnicas" que consisten en el asesoramiento en aspectos técnico-informáticos y en resolver todas aquellas dudas sobre el funcionamiento virtual del curso.

En segundo lugar la macrocategoría "Funciones por fases" en la que se pone el acento en la cronología del rol desarrollado por el tutor y en la planificación organizada de su trabajo. Las fases se organizan de manera cronológica: en una primera fase se realiza la "planificación del curso", es decir, se planifican todos los aspectos didácticos, logísticos y administrativos, la segunda fase o "fase de inicio", en la que el tutor deberá actuar como un perfecto anfitrión virtual. En esta fase se debe dar la bienvenida a los estudiantes, presentarse y promover que todos los estudiantes se presenten y comiencen a interactuar. Es el momento que se aprovecha para introducir el curso presentando y 
comentando los objetivos y el plan del curso. La siguiente fase representa la verdadera inmersión en el curso a realizar. Es la denominada "fase de desarrollo". En ella el tutor desarrolla diversas funciones pedagógicas como la orientación y la motivación, y tiene carácter organizativo-administrativo. Finalmente se produce la "fase de clausura". En esta fase se van finalizando y cerrando todas las tareas abiertas y se atiende especialmente a aquellos estudiantes que todavía tiene alguna tarea por realizar o finalizar.

La tercera macrocategoría que se presenta es la de "Funciones como Tareas". En cierta medida esta macro categoría es muy similar a la de "Funciones del tutor", pero poniendo el acento en las tareas y actividades que tiene que realizar el tutor sin organizarlas en funciones. Está compuesta por tres categorías: las "tareas más pedagógicas" en la que el tutor debe motivar, guiar y orientar y asesorar, es decir, además dinamizar y coordinar grupos, tiene que apoyar pedagógicamente y fomentar el aprendizaje colaborativo, la categoría "tareas más técnicas" que incluye tareas como las de informar del plan de trabajo, controlar la información y comunicación, realizar una atención rápida y adecuada, y una tercera, en la que el tutor debe realizar también algunas "otras funciones" que tienen al mismo tiempo, carácter pedagógico y técnico como son las de elaborar informes individuales y de conjunto, realizar la evaluación y anticipar problemas.

Para finalizar, la cuarta macro categoría la denominaríamos como "Tipología de Tutor". Haría referencia a los diferentes roles que desempeñan los tutores. Así pues, se podría construir la tipología de "Tutor pedagógico" en el que prima las funciones y tareas pedagógicas por encima de otro tipo de funciones, en segundo lugar estaría el "Tutor técnico" que prioriza las tareas y acciones técnicas en su trabajo de atención al estudiante, la tercera tipología sería la del "Tutor fases" en el que da una especial importancia a la planificación y el orden de las tareas para un trabajo de tutoría organizado. Por último, el "Tutor tareas" es el que ha de realizar una serie de acciones (tareas o actividades) para cumplir con su trabajo de asesoramiento.

En la siguiente figura se muestra, a modo de ejemplo, un gráfico en el que se representa esta última macro categoría. En ella se puede observar que en la tipología de "Tutor pedagógico" se podrían encuadrar los tutores 1, 2, 3, 4, 5 y 7. Su trabajo se organiza en función de la atención pedagógica del estudiante. En segundo lugar estaría el "Tutor técnico" en el que se encuadrarían los tutores 6, 7 y 8. Además se podrían destacar las tipologías de "Tutor fases" y "Tutor tareas", representadas por un tutor en cada caso: el 6 y el 8, respectivamente.

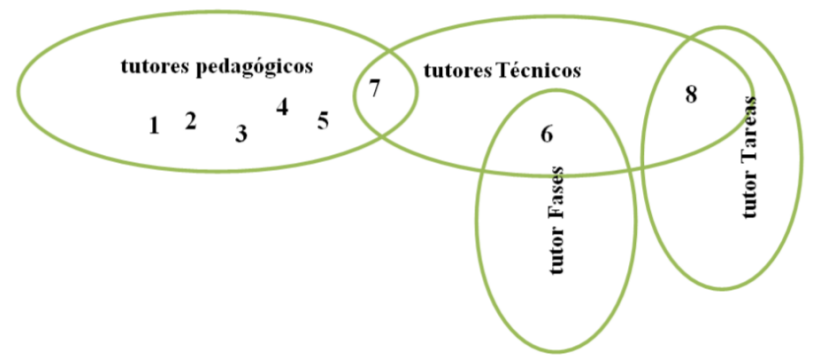

Figura 1. Representación gráfica de la macro categoría "tipología de tutor" 


\section{Discusión y conclusiones}

A través de este trabajo, se ha podido diseñar y elaborar un instrumento para estudiar las funciones puestas en práctica por los tutores durante su labor de tutorización en los cursos de e-learning, y se han llegado a conocer las percepciones que tienen los estudiantes sobre estas funciones.

El instrumento ha demostrado conseguir una medida suficientemente fiable y válida, una adecuada validez de contenido (reflejando adecuadamente las variables y actividades del teletutor), y de validez de constructo (instrumento coherente con la estructura teórica con la que fue diseñado).

El hecho de que las respuestas de los estudiantes, sobre cada una de las tareas incluidas en las funciones, hayan sido muy similares, sirve como confirmación también de la adecuación del uso de este tipo de instrumentos para conocer el perfil desarrollado por cada tutor, según las funciones puestas en práctica a partir de las opiniones de sus estudiantes.

En este trabajo, además, se han podido verificar y comprobar algunos de los aspectos que habían sido destacados por varios autores.

Schneeberger (2006) estableció que en las personas hasta los 35 años predominan las razones de promoción o la de mejorar en su trabajo. En cambio, en aquellas con edades por encima de los 35 años, destaca la razón de la "formación funcional de adaptación para mantener la empleabilidad de su profesión", o como se puede señalar también, para mejorar su curriculum profesional. Los resultados obtenidos por Schneeberger (2006) se han confirmado en este estudio. Los resultados señalan además que los trabajadores que realizan formación, tienen muy claramente definidos el objetivo y la idea de por qué quieren realizarla.

A partir de estas afirmaciones se puede concluir también, que se ha podido conocer cuáles son los motivos de los estudiantes para solicitar este tipo de cursos. Dicho estudio descriptivo arrojó además como resultados, que las causas de abandono de la formación por parte de los estudiantes podría deberse a "la falta de tiempo" para realizarla, a que "los contenidos no se correspondieran con sus expectativas", a que "el tutor no les motivara ni incentivara lo suficiente" durante la realización del mismo o a que "una vez comenzado el curso no se encontrara motivado haciéndolo". Dichas causas mencionadas fueron ya consideradas por García Teske (2007) como determinantes de las renuncias a seguir con la formación por parte del alumnado.

Por otro lado, los estudiantes afirman que no dejarían la formación por "tener poco dominio del entorno virtual de formación", ni por "no tener un buen dominio de la informática" para poder realizar el curso, o "por tener problemas de acceso a Internet o a un ordenador para su realización". Todo ello podría ser de debido a que los estudiantes consideran estas causas como subsanables, y con menos importancia que las anteriormente señaladas, mostrando así un verdadero interés por la formación en sí, por encima de posibles obstáculos que pueden surgirles, considerando que pueden ser solucionados por ellos mismos. No obstante, se debería indagar más sobre estos aspectos para poder obtener unas conclusiones más certeras y poder así establecer el motivo de esas conclusiones. 
Tras realizar el estudio descriptivo, se ha podido comprobar con los resultados obtenidos, que casi la totalidad del alumnado considera que está cubriendo sus expectativas. En este sentido, Marcelo (2011) establece que aunque la evaluación de la formación, a través de la satisfacción de los estudiantes, es una medida necesaria pero no suficiente para poder comprender toda la complejidad que supone una acción formativa, sí se puede considerar como un valor relevante para conocer las variables que más influyen en la percepción de calidad por parte de los mismos.

El análisis de los datos refleja que las funciones organizativa, académica y orientadora, por este orden, son las variables mejor evaluadas, siendo la técnica y la social las que obtienen una menor valoración. Esto quiere decir que según la opinión del alumnado, los tutores han desempeñado, de manera más clara, la labor de guiar y asesorar al alumnado durante el desarrollo de las acciones formativas, han tenido las competencias adecuadas para el dominio de los contenidos, actividades, diagnóstico y evaluación formativa del alumnado, han desarrollado habilidades para organizar las actividades, y han sido capaces de planificar, estructurar, establecer las normas de funcionamiento, tiempos, calendarios y puesta en marcha de las distintas acciones formativas.

En cambio opinan que, en menor medida, se han asegurado de que los estudiantes sean capaces de poseer cierto dominio sobre las herramientas disponibles en el entorno virtual de aprendizaje y de sus herramientas de comunicación, así como, evitado los sentimientos de aislamiento, pérdida o falta de motivación del alumnado durante las acciones formativas tutorizadas.

A través del análisis de los datos del cuestionario para comprobar en qué funciones concretas se producían diferencias en los distintos tutores, se pudo comprobar que éstas eran escasas. Esto posiblemente tenga que ver con que los tutores pertenecían a una misma empresa, habían recibido las mismas directrices y similar formación sobre tutorización e-learning.

En la fase cualitativa de la investigación también se han obtenido conclusiones de relevancia. Por ejemplo, se ha evidenciado la adecuación de la perspectiva fenomenográfica adoptada, a partir del uso de las entrevistas a tutores y la posterior ampliación de información, como una herramienta que ha puesto de manifiesto las categorías y dimensiones principales. El análisis cualitativo realizado ha permitido además organizar los diversos sistemas de categorías en otros tantos "marcos teóricos", representados a través de diagramas comprensivos, que nos ayudan a entender de manera más adecuada las relaciones entre categorías y dimensiones en los tutores del estudio.

El análisis cualitativo de las entrevistas y de los documentos ha permitido indagar en las concepciones de la función docente, pudiendo llegar incluso a establecer tipologías de tutores según las funciones y roles desarrollados, los recursos más utilizados en su labor de tutorización y los rasgos que caracterizan a los estudiantes que realizan este tipo de formación. Se ha considerado, por tanto, también una adecuada estrategia cualitativa, para construir sistemas de categorías, para estudiar y profundizar en las categorías preestablecidas (deductivas), y para construir otras nuevas categorías y subcategorías (inductivas o ad hoc) con las que analizar, en este y en futuros estudios, las funciones y roles desempeñados por los docentes. 
Así pues, a través de las macro categorías, categorías y subcategorías elaboradas ad hoc se ha propuesto un diagrama comprensivo que nos ha ayudado a entender la manera en que los tutores realizan su labor docente en los cursos de formación e-learning, así como el contexto formativo en el que se desarrolla. Así mismo, la creación de estas macro categorías con sus respectivas categorías y subcategorías, ha servido para poder elaborar modelos de tutoría en e-learning que faciliten conocer las relaciones de interacción en el proceso de tutoría.

\section{Referencias bibliográficas}

Aballay, L., Aciar, S., González, C., \& Collazos, C. (2016). Método de Medición de la Percepción de los usuarios sobre los sistemas E-Learning de los Centros e Instituciones Educativas. International Journal of Information Systems and Software Engineering for Big Companies (IJISEBC), 3(1), 28-42. Recuperado de http://www.uajournals.com/ ojs/index.php/ijisebc/article/view/123/110

Cabero, J. (2013). El aprendizaje autorregulado como marco teórico para la aplicación educativa de las comunidades virtuales y los entornos personales de aprendizaje. Revista Electrónica Teoría de la Educación: Educación y Cultura en la Sociedad de la Información. 14(2), 133-156. Recuperado de http://www.redalyc.org/pdf/2010/201028055006. pdf

Cabero, J., \& Llorente, M.C. (2010). Comunidades virtuales para el aprendizaje. EDUTEC, Revista Electrónica de Tecnología Educativa, 34, 1-10. doi: http://dx.doi.org/10.21556/ edutec.2010.34.419

Curci, R. (2014). Satisfacción de los estudiantes respecto a las acciones formativas e-learning en el ámbito universitario. Pixel-Bit. Revista de Medios y Educación, 44, 215-229. doi: http://dx.doi.org/10.12795/pixelbit.2014.i44.15

Fandos, M., \& Cano, J. (2013). Formación a distancia y retos actuales en los roles docentes y su vinculación con la empresa: propuesta y controversias. Revista Electrónica de Tecnología Educativa, 45, 1-13. doi: http://dx.doi.org/10.21556/edutec.2013.45.14

Fernández-Jiménez, M.A., Tójar-Hurtado, J.C., \& Mena-Rodríguez, E. (2013). Evaluación de buenas prácticas de tutorización e-learning. Funciones del teletutor y su papel en la formación. Píxel-Bit. Revista de Medios y Educación, 43, 85-98. doi: http://dx.doi. org/10.12795/pixelbit.2013.i43.08

Fernández Robles, B., \& Cabero, J. (2016). Percepciones de teleformadores del Instituto de Formación y Estudios Sociales de Sevilla sobre la teleformación. Innoeduca. International Journal of Technology and Educational Innovation, 2(1), 4-12. doi: http:// dx.doi.org/10.20548/innoeduca.2016.v2i1.1218

Fidalgo, Á., Sein-Echaluce, M.L., Lerís, D., \& Castaneda, O. (2013). Teaching Innova Project: the Incorporation of Adaptable Outcomes in Order to Grade Training Adaptability. Journal UCS, 19(11), 1500-1521. doi: http://dx.doi.org/10.3217/jucs-019-11-1500

García Teske, E. (2007). El "abandono” en cursos de e-learning: algunos aprendizajes para nuevas propuestas. Revista Iberoamericana de Educación, 44(3), 1-16. Recuperado de https://dialnet.unirioja.es/servlet/articulo?codigo $=2471855$ 
Llorente, M.C. (2006). El tutor en E-learning: aspectos a tener en cuenta. Eductec. Revista electrónica de Tecnología Educativa, 20. Recuperado de http://edutec.rediris.es/Revelec2/ revelec20/llorente.htm.

Llorente, M.C. (2005). La tutoría virtual: técnicas, herramientas y estrategias. Conferencia presentada en Eduweb (Valencia-Carabobo-Venezuela). Recuperado de http://tecnologiaedu.us.es/bibliovir/pdf/tutoriavirtual.pdf.

Marcelo, C. (2011). E-learning en la formación para el empleo: ¿qué opinan los usuarios?. Revista de Educación, 355, 285-308.

Picón, M. (2013). Guía de tutorización e-learning. Orientaciones básicas sobre la docencia en modalidad de teleformación. Ingeniería e Integración Avanzadas (Ingenia). Instituto Andaluz de Administración Pública Sevilla: Instituto Andaluz de Administración Pública. Junta de Andalucía. Recuperado de http://www.juntadeandalucia.es/ agenciadecalidadsanitaria/formacionsalud/export/sites/default/galerias/aportesDocumentos/pildora/1371807956935.pdf

Reigeluth, C. (2012). Teoría instruccional y tecnología para el nuevo paradigma de la educación. RED, Revista de Educación a Distancia, 32. Recuperado de https://www. um.es/ead/red/32

Rodríguez, N. (2014). Fundamentos del proceso educativo a distancia: enseñanza, aprendizaje, evaluación. Revista Iberoamericana de Educación a distancia, 17(2), 75-99.

Sánchez López, M.E, \& Gallego, A. (2014). La función tutorial ante el reto de la enseñanza online: algunas experiencias. Docencia y Derecho, Revista para la docencia jurídica universitaria, 8. Recuperado de https://www.uco.es/docencia_derecho/index.php/ reduca/article/viewFile/84/107

Schneeberger, A. (2006). Cualificados para la Sociedad del Conocimiento y de los Servicios. Tendencias que determinan la futura demanda de forma inicial y continua. Revista Europea de Formación Profesional, 38(2), 6-25. Recuperado de www.oei.es/etp/ cualificados_sociedad_conocimiento_servicios.pdf

Volles, N. (2016). Lifelong Learning in the EU: Changing Conceptualisations, Actors, and Policies. Studies in Higher Education, 41(2), 343-363.

Fecha de recepción: 31 de octubre de 2016

Fecha de revisión: 31 de octubre de 2016

Fecha de aceptación: 15 de febrero de 2017 